\title{
Appreciating the global impact of cleft lip and palate at the medical school level
}

\author{
Youssef Tahiri, Saoussen Salhi, Hani Sinno, Mario Luc
}

\section{INTRODUCTION}

Medical schools take on the responsibility to teach future physicians the basics of cell biology, biochemistry, physiology, genetics, pathology, pharmacology and during the last years, introduce medical students to the clinical application of these subjects.

However, at the end of medical school, most, if not all students are unable to appreciate the global, societal and personal impact diseases and illnesses can have.

Orofacial clefts represent the second most frequent congenital anomaly and the most common congenital anomaly at the level of the craniofacial region $(1,2)$. An orofacial cleft can affect the lip, or palate or both, and can be unilateral or bilateral, depending on which step of facial development has been disrupted (3). Worldwide, the problem is significant with an incidence that ranges from 1:500 to 1:2500 children each year, varying with geographic location, ethnic group and socioeconomic conditions (1). This problem is indeed more common in developing countries, especially those in Asia with an incidence of 1:500 (3), compared to an incidence of $1: 800$ in the United States (4). The reason for this discrepancy remains unknown, but it is hypothesized that the lack of prenatal care, genetic inheritance and poor nutrition are all contributing factors (5).

This article doesn't aim at being exhaustive on the subject of cleft lip and palate. Rather, it aspires at inviting medical students to gain an awareness of the global impact of orofacial clefts. First, we will provide a brief overview of the pathophysiology of clefts and expand on its epidemiology and the humanitarian organizations involved in correcting clefts on a global scale. Then, we will present the areas that, in our view, need improvement to ultimately correct more clefts in the best way possible.

\section{PATHOPHYSIOLOGY AND ETIOLOGY OF CLEFT LIP AND PALATE}

What is a cleft lip or palate? A simple way to explain it would be that it is a congenital malformation that starts as a disruption in the foetal development resulting in an abnormal anatomy and function at birth.

Embryologically speaking, the development of the face takes place between the 4th and 10th weeks of gestation. It occurs by the development and fusion of five prominences: the frontonasal prominence, the two maxillary prominences and the two mandibular prominences (6).

A cleft lip has been attributed to failure of the mesenchyme of the maxillary prominence and medial nasal processes (derived from an invagination on the frontonasal prominence) to develop adequately (6). This hypoplasia of the maxillary prominence and two nasal processes subsequently results in failure of their fusion (6). This fusion normally leads to the formation of the intermaxillary process which later develops into the philtrum and primary palate containing the four incisors (6). Therefore, the abnormal fusion can lead to a cleft that may range in length from a minor notch in the vermilion border of the lip, just lateral to the philtrum, to a cleft that completely separates the lateral lip from the philtrum and nasal cavity (6). The depth of clefting also varies: a cleft can involve just the soft tissue of the lip or divide the lateral portion of the maxillary bone from the premaxillary portion (that bears the incisors). These latter clefts result in deformed, absent or supernumerary teeth (6).

On the other hand, the palate development starts around the 7th week of gestation, when the medial 
walls of the maxillary prominences produce a pair of thin medial extensions called the palatine shelves. These shelves first grow downward and at the end of the 7th week, rotate upward into a horizontal position before fusing with each other and with the primary palate to form the secondary palate. Failure of these palatine shelves to fuse results in a cleft palate (6).

The etiology of these two anomalies appears to be multifactorial. First, if a parent was born with a cleft, the offspring will in turn be affected in $7 \%$ of cases. Moreover, if in addition to a parent, there is also a sibling affected, then the child will have a cleft in $14 \%$ of instances (2). This reflects the genetic inheritance of these anomalies. However, if a child suffers from a cleft, the chance of his monozygotic twin also having a cleft is only $60 \%$. This suggests that there are other nongenomic factors (3). Some of these include environmental factors, such as drugs (valproic acid, thalidomide, phenytoin...), maternal alcohol and tobacco use, dioxins and other herbicides, and possibly high altitude (3). Chromosomal and nonchromosomal syndromes are also associated with clefting (e.g. Stickler and Treacher Collins syndromes) $(3,6)$.

The treatment of cleft lip and palate is surgical closure. The timing of cleft lip repair is controversial, but the general agreement is to perform the repair between 6 weeks and 9 months of age. The ultimate goal of the repair is to establish symmetrical nostrils, alar bases, natural philtrum columns and central dimple, as well as the Cupid's bow and vermilion tubercle (2).

In terms of cleft palate repair, many surgeons now prefer earlier closure by 6-12 months of age to preserve adequate speech development. The various cleft palate repairs are designed to reorient the musculature of the palate, close the cleft and lengthen the palate (2).

\section{IMPACT ON A GLOBAL SCALE}

A cleft palate is associated mostly with complications relating to speech. A competent palate is necessary for normal speech production. It elevates and meets the posterior pharyngeal wall during speech and also during swallowing. A cleft palate impairs this function causing the speech to become hypernasal or incomprehensible, as well as difficulty in swallowing (2). As mentioned above, an early repair is essential for normal speech development. Indeed, a later repair is associated with a more difficult regain of normal speech function (7).
Moreover, in patients with a cleft palate, the muscles of the palate are abnormally arranged around the Eustachian tube's opening into the pharynx causing recurrent otitis media. Almost all of these children would require myringotomy tubes to avoid long-term hearing problems (2).

A cleft lip, on the other hand, is usually not associated with any such complications. The "only" reason why a cleft lip is repaired is for the sake of appearance (2). It may certainly seem like a trivial reason to not only perform surgery on a child but also mobilize resources to perform it on children of developing countries where other problems may seem more urgent and worthy of those resources.

An orofacial cleft results in disfigurement that alone can cause affected children to be socially isolated especially by their peers in school (7). Furthermore, as mentioned above, the clefts occur with a higher incidence in developing countries, where it is not unusual to encounter strong cultural and religious beliefs especially in the most remote villages (7). For instance, in Togo where Voodoo remains an important belief system, it is seen as a curse to be born with a cleft. If the cleft is too disfiguring, it may be decided to sacrifice the child (7). Thus, it seems that in the best case scenario, a child with a cleft ends up as a social outcast, hidden away by his family, and does not attend school either because he cannot speak properly or because he is rejected by his peers (7).

\section{HUMANITARIAN ORGANIZATIONS}

A few humanitarian organizations travel to developing countries to attend to children with craniofacial deformities including orofacial clefts. The most popular of these organizations is probably Operation Smile International (OSI). This is a non-governmental organization that provides reconstructive surgery for children and young adults around the world born with cleft lips, palates and other craniofacial deformities (8). It was founded in 1982 by a plastic surgeon Dr. William Magee Jr. alongside his wife Kathleen, a nurse and clinical social worker $(8,4)$. Since then, OSI has provided free surgeries to more than 140,000 children in 50 countries (9). A medical mission team assembles plastic surgeons, anaesthesiologist, nurses, a paediatrician, a dentist, a speech pathologist, a child life specialist and a biomedical technician (8). In a typical two-week mission, from 300 to 500 children are screened to determine whether or not they are candidates for repair (8). If a child is too young, malnourished or is otherwise judged unable 
to sustain the stress of surgery and anaesthesia, he/she will not be operated on (10). Furthermore, the cases requiring prolonged anaesthesia and complicated postoperative follow-up are also avoided since a postoperative team remains at the host site only for three days (5). In the end, about 100 to 150 children are surgically treated. Each surgery takes from 45 to 60 minutes (8)

The team brings along supplies, instruments and equipment. However, the host sites are required to provide specific necessities such as oxygen, beds, electricity, and water. Moreover, the hosts are asked to advertise to the countryside about the patient screening. This is most commonly accomplished through schools, churches and mission groups since mass media is often absent (5).

An important ally to OSI is The Flying Hospital Inc., another non-profit humanitarian organization. It is a jumbo jet equipped with a twelve-bed preoperative and postoperative suite, three-position surgical suite, two-position dental, ophthalmologic, and otorhinolaryngologic areas, sterilization station, nurses' station, and scrub area. It also contains on-board oxygen generation, nitrous oxide, a medical air system, medical vacuum, and a water purification system. The jet's lower level houses a pharmacy, patient check-in and waiting areas that also serve as classrooms for patient education and on-site physician training (4).

Another goal of OSI is for the on-site health care professionals to learn the procedures and become familiar with the new equipment brought along by the visiting team. Dr. Magee indeed explained "it is our hope that, after we've left, the medical training and technology donations will help people in these countries become more self-sufficient" (4).

Nevertheless, this aspect of OSI could be seen as an area to improve (7). Indeed, Dr. Pavi, a paediatric plastic surgeon, member of another nongovernmental organization (NGO) called "la chaîne de l'espoir", pointed out the "flaw" of OSI. They bring with them new equipment and their own jet fully equipped, teach the on-site doctors how to use the new equipment and then leave with their jet. If the host sites run out of the equipment brought along by the OSI team, the hosts are back to square one, where they don't have the capacity to operate on children with orofacial clefts (7).

Unlike OSI, "la chaîne de l'espoir" is a smaller NGO. On a mission to Togo that lasted two weeks, Dr. Pavi and Dr. J.Moren, an anaesthesiologist, performed four surgeries per day, amounting to a number of about 40 children operated on for cleft lip or palate. This number is much smaller than the OSI one. However, when Dr. Pavi and Dr. Moren arrived in Togo, they did not bring any equipment because their objective is to teach the on-site doctors how to perform the procedure with the equipment available at the host site. Thus, during each operation, Dr. Pavi teaches two on-site surgeons the procedure, with equipment that will be available to them even after the team has left the site (7).

\section{CHALLENGES TO IMPROVEMENT}

From what has been said before, it appears that there is significant room for improvement. A compromise between adapting the teaching of the host-site health care professionals to the type of equipment that is available to them and operating on a maximum of children has to be reached. Indeed, the ultimate goal of these types of organizations should be to make their help no longer a necessity to developing countries which should become autonomous in their capacity to operate on children with orofacial clefts (7).

However, even if the on-site surgeons acquire the expertise, there is still the problem of the cost of these types of surgeries. In Togo for example, a single cleft lip or palate repair costs about 450 dollars, exceeding the monthly salary of the surgeon performing the procedure (7). So why is the surgery so expensive? The answer is that in developing countries such as the countries of West Africa, in order to have a surgery, a patient must pay for all the supplies needed including the antibiotics, the compresses, the sterilizing agents... Moreover, even if the patient is able to afford those supplies, the pharmacies often lack them (7). Therefore, it seems that as long as developing countries have poor economies, the NGOs mentioned above will be needed, if only to help in terms of funding.

Another problem faced by developing countries is that doctors from these countries usually leave to study medicine in Europe or North America. There they have access to well-equipped hospitals and the newest medical technologies and therefore seldom return to their home countries where they are needed the most. These surgeons should be encouraged to return home once their medical training is completed or at least actively participate in humanitarian missions (7).

Furthermore, as with any surgery, there can be complications associated with cleft lip or palate repair. Identification and correction of these complications would require follow up for longer than what the teams spend in each country. Team 
participants should be audited for their performance and a system that measures surgical outcomes should be implemented (1). Such a system should take into consideration the degree of disability that persists despite or as a result of the surgery: defects in nasolabial appearance, palate integrity (fistulas) and function (speech and swallowing), hearing, dental development and psychosocial adaptation (1). Among all of these factors, nasolabial appearance is the only outcome that can be properly and reliably assessed within 1 year of the operation. The other elements would require a longer followup period (1). This outcome measurement was thought of by OSI and attempted during the "World Journey of Smiles" in November 2007: during a period of 10 days, at 40 simultaneous mission sites, high quality digital images were collected during screening and under anaesthesia before surgery, immediately after surgery while still under anesthesia and at a 1-week, 6-month, and 1-year follow-up visits organized by local foundations. With these elements, an outcome evaluation chart was created for each patient (1). A plastic surgeon member of the International Outcomes Council scored the results using a standardized evaluation system. The final feedback reports were sent to respective surgical teams, in-country executive and medical directors as well as to each surgeon listing which patients they had operated on (1).

This is a major advancement that should be added to any NGO. Indeed, it is only fair that their activities be reported not only by the number of procedures performed but also procedural outcomes. However, quality images can only assess symmetry and aesthetics. Indeed, the current system is still unable to evaluate speech, hearing and feeding abilities postoperatively. Moreover, there remains the problem that the majority of patients do not come back for follow-up visits. This is often due to their inability to travel to the follow-up sites mostly because they generally live in remote geographical areas. Parents also tend to think that a follow-up visit is unnecessary if they do not see a complication. A way to address this last obstacle is to stress to parents, during their first visit, the importance of follow-up as a way to identify those children who need further treatment to complete their care (1).

Finally, NGOs could also add to their programs education for the inhabitants of the host countries regarding the meaning of orofacial clefting. This could be done by trained medical personnel brought along on missions. This could help affected children become accepted in the society they live in, even prior to surgical repair (4).

\section{CONCLUSION}

Orofacial clefting is a major problem worldwide, more prevalent in developing countries for poorly understood reasons. Unfortunately these countries lack the appropriate financial, medical, and technological resources to address this very common condition. As a result, several children worldwide are left to live with disfiguring facial malformations that cause them to quickly become social outcasts hidden away by their families.

Several humanitarian organizations travel to these countries to perform free operations on these children every year. Although the work accomplished is significant, there is still room for improvement when one looks at the number of children who receive the surgery versus the children who are turned down for reasons mentioned earlier.

To teach the on-site surgeons how to operate on cleft lips and palates with on-site equipment rather than new expansive supplies that the host countries will never be able to afford, should become a primordial priority to these NGOs. Moreover, surgeons from developing countries, who train in developed countries, should be encouraged to return home after their training is completed in order to make their knowledge and expertise available to children who need it most.

Furthermore, a system to evaluate surgical outcomes should be implemented by these NGOs for two reasons: first, so surgeons can receive feedback on their operating skills and improve on them accordingly and second so children who require additional treatment can be identified.

Despite all of this, developing countries will still need financial support to afford the basic supplies needed for surgeries (compresses, antibiotics...).

Meanwhile, the help of these NGOs is still needed, and it seems that a simple way to have more children operated on during a mission is to have more people participate in these missions. Therefore, we encourage all interested medical students and residents to participate either in their future careers or in academic activities to help improve the care of orofacial clefting worldwide.

\section{REFERENCES}

1. Bermudez L, Carter V, Magee W, Sherman R, Ayala R. Surgical outcomes auditing systems in humanitarian organizations. World journal of surgery. 2009 Oct 17:1-8.

2. Wirth GA, Evans GRD. Plastic Surgery: Diseases of the Skin and Soft Tissue, Face, and Hand. In: Lawrence PF, Bell RM, Dayton MT, Ahmed MI, Hebert JC, editors. Essentials of Surgical Specialties. Philadelphia, PA: Lippincott Williams \& Wilkins; 2007. p. 138-140.

3. Bishop WP. The Digestive System: Oral Cavity. In: Kliegman RM, Jenson HB, Marcdante KJ, Behrman 
RE, editors. Essentials of Pediatrics. Philadelphia, PA: Elsevier Saunders; 2006. p. 595.

4. Phillips DF. Operation smile volunteers travel far to transform lives. JAMA. 1999 Feb 17; 281(7): 597-598.

5. Poe D. Operation smile international: missions of mercy. Plastic surgical nursing. 1994 Winter; 14(4): 225-230.

6. Francis-West PH. Development of the Pharyngeal Apparatus and Face. In: Schoenwolf GC, Bleyl SB, Brauer PR, Francis-West PH, editors. Larsen's human embryology. Philadelphia, PA: Churchill Livingstone Elsevier; 2009. p. 563-571.
7. Metivet JP, Stelletta P, Moyon C, Lemoine G. Les visages sans sourire [documentary]. France : Envoye Special; 2009.

8. Soloveoff $\mathrm{S}$. Operation smile giving the gift of a smile. Plastic surgical nursing. 2005 Jul-Sep; 25(3):122-123.

9. Operation smile [Internet]. Norfolk: Operation Smile International; 2009. Who we are; 2009 [cited 2010 Jan 18]; [about 2 screens]. Available from: http://www. operationsmile.org/about_us/who-we-are/

10. Samson G. Operation smile-China. Canadian operating room nursing journal. $2003 \mathrm{Dec} ; 21(4): 13-17$.

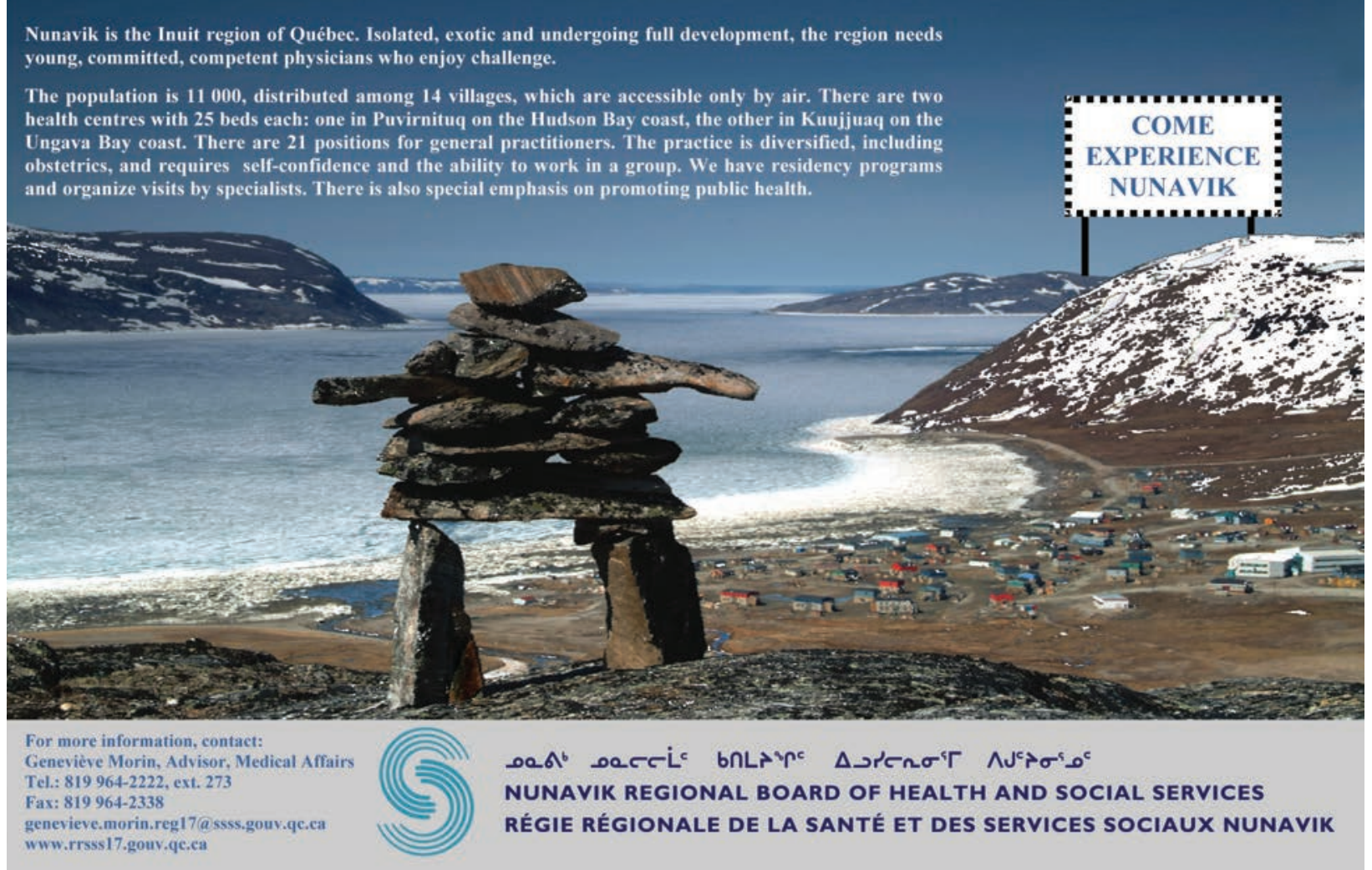

